\title{
Cytokines Released from Allergen- Stimulated Blood Cells during the Delayed Asthmatic Response to Allergen Challenge
}

\author{
Zdenek Pelikan \\ Allergy Research Foundation, Breda, The Netherlands \\ Email: zpelikan@casema.nl
}

Received 13 January 2014; revised 10 February 2014; accepted 9 March 2014

Copyright (C) 2014 by author and Scientific Research Publishing Inc.

This work is licensed under the Creative Commons Attribution International License (CC BY). http://creativecommons.org/licenses/by/4.0/

(c) (i) Open Access

\section{Abstract}

Background: Bronchial asthma patients may develop various asthmatic response types to bronchial challenge with allergen, such as immediate (IAR), late (LAR), dual (DAR) or delayed (DYAR), due to different immunologic mechanisms. The DYAR, beginning between $26-32 \mathrm{hrs}$ and lasting up to $56 \mathrm{hrs}$ after the allergen challenge, differs from the IAR, LAR and DAR in clinical, diagnostic and immunologic aspects. The aim of this study was to investigate the concentrations of the particular intracellular cytokines released by blood cells stimulated with relevant allergens "in vitro", before and during the DYAR. Methods: In 23 patients, the repeated DYAR $(p<0.001)$ was supplemented with cytokine determination in the supernatants of the blood cells stimulated with relevant allergens before and up to 72 hours after the bronchial challenge, by means of enzyme-linked immunoassay. Results: The significantly elevated pre-challenge concentrations $(p<0.05)$ of IL-2, IL-17, IFN- $\gamma$ and G-CSF released by allergen-stimulated blood cells "in vitro" were recorded in the DYAR patients as compared with healthy controls. The significantly increased post-challenge concentrations $(p<0.05)$ of IL-1 $\beta$, IL-2, IL-8, IL-12p70, IL-18, IFN- $\gamma$ and TNF- $\alpha$, whereas decreased concentrations of IL-4, IL-6 and IL-17, were released by blood cells stimulated with relevant allergens "in vitro", as compared both with their pre-challenge concentrations and with the corresponding PBS control values. Conclusions: The profiles of cytokines released by allergen-stimulated peripheral blood cells during the DYAR would suggest an activation of $\mathrm{Th}_{1}$ cells, neutrophils, monocytes and probably also bronchial macrophages, epithelial and endothelial cells and their involvement in the immunologic mechanism(s) underlying the clinical DYAR.

\section{Keywords}

Delayed Asthmatic Response; Cytokines; Allergen-Stimulated Peripheral Blood Cells 


\section{Introduction}

In allergic bronchial asthma various immunologic mechanisms can be involved [1]-[8]. The causal role of immediate hypersensitivity upon participation of IgE antibody, mast cells/basophils, eosinophils and Th2-lymphocytes, in this condition, has already been established [1]-[8]. Although, some evidence for participation of the other immunologic mechanisms has already been gathered, our knowledge of the involvement of the non-IgE mechanisms in the bronchial asthma remains still incomplete [1] [5]-[11]. Patients with bronchial asthma being challenged with allergens may develop different asthmatic response types, such as immediate (IAR), late (LAR) or dual (DAR) response, having been already studied from various points of view [3] [5] [7]-[22]. Recently, we have described an asthmatic response appearing between 26 - 56 hours after the bronchial challenge with various inhalant allergens, designated as a “delayed asthmatic response” (DYAR) [23] [24]. This response type exhibited different clinical, immunologic and pharmacologic features from the IAR, LAR and DAR [12] [13] [23] [24]. The purpose of this study, was to investigate: 1) the cytokine profiles released by peripheral blood cells of patients developing the DYAR after the "in vitro" stimulation with relevant allergens; 2) the possible changes in the particular cytokines released bythe "in vitro" stimulated blood cells with allergen during the DYAR; 3) the activation degree of particular blood cell types during the DYAR.

\section{Material and Methods}

\subsection{Patients}

Twenty-three asthmatics examined at our Department of Allergology \& Immunology, Inst. Med. Sci. "De Klokkenberg”, Breda, The Netherlands during a period 1998-1999 and demonstrating DYAR after the bronchial allergen challenge (Figure 1) volunteered to participate in this study. These patients, 20 - 51 years of age, suffered from reversible bronchial constriction alternating with symptom-free periods, but without any restrictive changes of their pulmonary function (Table 1). They did not use oral corticosteroids or immunotherapy and had no airway infections. They were examined by routine diagnostic procedure, serving also as inclusion-exclusion criteria, including various diagnostic parameters (Table 1), among others also 47 bronchial provocation tests with inhalant allergens (BPT) (Table 2) and 23 phosphate buffered saline (PBS) control challenges. All BPTs were performed in a period without manifest bronchial complaints, outside the allergen-relevant season and during hospitalization. Inhalation corticosteroids $(\mathrm{n}=9)$ and long-acting $\beta 2$-sympathomimetics $(\mathrm{n}=5)$ were

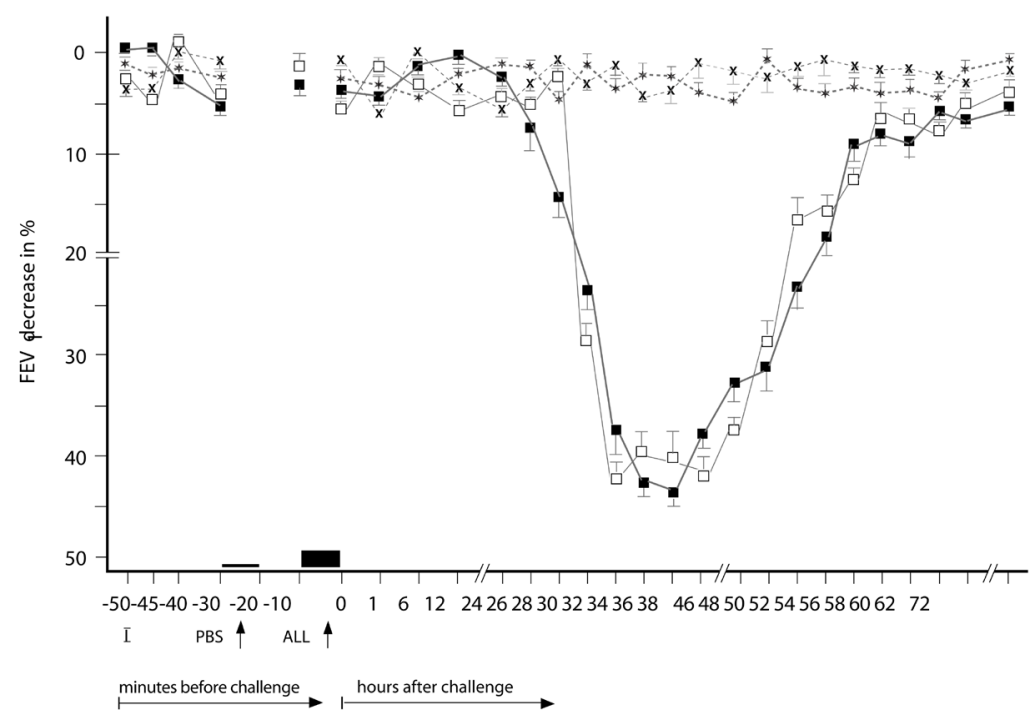

Figure 1. Delayed asthmatic response to allergen challenge (DYAR) and phosphate buffered saline (PBS) control challenge. The mean percentage changes in the $\mathrm{FEV}_{1}$ values calculated from 23 DYARs and 23 PBS control challenges; ( $\left.\square\right)$ $=$ the initial DYAR; $(\square)=$ the repeated DYAR; $\left({ }^{*}\right)=$ the initial PBS; $(x)=$ the repeated PBS; I = initial (baseline) values; ALL = allergen challenge; PBS = phosphate buffered saline; Bars = means \pm SEM. 
Table 1. Characteristics of the patients and control subjects.

\begin{tabular}{|c|c|c|c|c|c|}
\hline & \multicolumn{5}{|c|}{ Control subjects } \\
\hline & \multirow{2}{*}{$\begin{array}{c}\text { Patients } \\
\text { DYAR n = } 23\end{array}$} & \multicolumn{3}{|c|}{ Patients with asthma } & \multirow{2}{*}{$\begin{array}{l}\text { Healthy subjects } \\
\qquad \mathrm{n}=25\end{array}$} \\
\hline & & IAR $n=24$ & $\mathrm{LAR} n=16$ & $\mathrm{DAR} \mathrm{n}=17$ & \\
\hline Age (years) & $27 \pm 8$ & $30 \pm 4$ & $29 \pm 6$ & $28 \pm 4$ & $30 \pm 7$ \\
\hline Gender (M/F) & $11 / 12$ & $10 / 14$ & $7 / 9$ & $9 / 8$ & $12 / 13$ \\
\hline Disease history (years) & $4.8 \pm 2.2$ & $4.5 \pm 2.7$ & $5.1 \pm 2.1$ & $4.8 \pm 2.9$ & 0 \\
\hline Asthmatic attacks per month & $3 \pm 1$ & $4 \pm 1$ & $3 \pm 2$ & $3 \pm 2$ & 0 \\
\hline FEV 1 (\% predicted) & $95.2 \pm 6.1$ & $97.5 \pm 6.3$ & $97.2 \pm 3.5$ & $99.2 \pm 3.1$ & $104.3 \pm 2.5$ \\
\hline FVC (\% predicted) & $98.7 \pm 4.1$ & $98.3 \pm 3.3$ & $99.3 \pm 2.0$ & $98.2 \pm 4.4$ & $103.4 \pm 5.3$ \\
\hline Blood leukocyte count $\left(\times 10^{9} / \mathrm{L}\right)^{\circ}$ & $10.1 \pm 0.9^{+}$ & $6.3 \pm 1.1$ & $8.0 \pm 1.7$ & $8.2 \pm 0.7$ & $7.1 \pm 0.5$ \\
\hline Blood eosinophil count $\left(\times 10^{6} / \mathrm{L}\right)^{\circ \circ}$ & $373 \pm 45$ & $569 \pm 71^{*}$ & $544 \pm 35^{*}$ & $551 \pm 53^{*}$ & $265 \pm 24$ \\
\hline Blood thrombocyte count $\left(\times 10^{9} / \mathrm{L}\right)^{\circ \circ 0}$ & $324 \pm 58$ & $311 \pm 24$ & $346 \pm 35$ & $279 \pm 39$ & $306 \pm 28$ \\
\hline \multicolumn{6}{|l|}{ Bronchial histamine threshold ${ }^{\square}$} \\
\hline$\leq 2.0 \mathrm{mg} / \mathrm{mL}$ & 2 & 3 & 2 & 3 & 0 \\
\hline $4.0 \mathrm{mg} / \mathrm{mL}$ & 1 & 4 & 1 & 2 & 0 \\
\hline $8.0 \mathrm{mg} / \mathrm{mL}$ & 2 & 6 & 5 & 3 & 0 \\
\hline $16.0 \mathrm{mg} / \mathrm{mL}$ & 7 & 3 & 3 & 4 & 0 \\
\hline $32.0 \mathrm{mg} / \mathrm{mL}$ & 5 & 5 & 3 & 3 & 1 \\
\hline$>32.0 \mathrm{mg} / \mathrm{mL}$ & 6 & 3 & 2 & 2 & 24 \\
\hline \multicolumn{6}{|l|}{ Positive skin response (i.c.) } \\
\hline Immediate & 8 & 21 & 5 & 10 & 0 \\
\hline Late & 2 & 3 & 11 & 7 & 0 \\
\hline Delayed & 13 & 0 & 0 & 0 & 0 \\
\hline Increased total IgE in serum" & 0 & 6 & 3 & 7 & 0 \\
\hline Positive specific IgE in serum" & 0 & 15 & 10 & 11 & 0 \\
\hline Increased total IgG in serum"' & 0 & 0 & 9 & 8 & 0 \\
\hline \multicolumn{6}{|l|}{ Increased IgG sub-classes in serum ${ }^{\Delta}$} \\
\hline $\operatorname{IgG}_{1}$ & 0 & 0 & 2 & 1 & 0 \\
\hline $\operatorname{IgG}_{2}$ & 0 & 0 & 0 & 1 & 0 \\
\hline $\operatorname{IgG}_{3}$ & 0 & 0 & 3 & 2 & 0 \\
\hline $\operatorname{IgG}_{4}$ & 0 & 0 & 5 & 2 & 0 \\
\hline Increased total IgM in serum ${ }^{\Delta \Delta}$ & 0 & 0 & 0 & 0 & 0 \\
\hline Increased total IgA in serum $^{\Delta \Delta \Delta}$ & 0 & 0 & 0 & 0 & 0 \\
\hline Ratio $\mathrm{Th}_{1} / \mathrm{Th}_{2}(\%)$ in blood ${ }^{\mathbf{}}$ & $8.5 \pm 2.1^{+}$ & $6.4 \pm 2.2$ & $6.6 \pm 2.0$ & $6.8 \pm 2.4$ & $7.0 \pm 2.3$ \\
\hline IFN- $\gamma(\mathrm{pg} / \mathrm{mL})-\mathrm{PBMC}^{\boldsymbol{\Lambda}}$ & $377 \pm 45^{+}$ & $219 \pm 30$ & $236 \pm 42$ & $198 \pm 51$ & $232 \pm 46$ \\
\hline IL-4 (pg/mL)-PBMC ${ }^{\boldsymbol{\Lambda}}$ & $17.1 \pm 6.3$ & $23.5 \pm 4.0^{+}$ & $21.6 \pm 4.0$ & $24.4 \pm 2.5^{+}$ & $16.8 \pm 3.0$ \\
\hline
\end{tabular}

DYAR = delayed asthmatic response; IAR = immediate asthmatic response; LAR= late asthmatic response; DAR = dual late asthmatic response; Values $=$ mean $\pm \mathrm{SD}$; Statistical significance as compared with healthy control subjects $={ }^{+} p \leq 0.05,{ }^{*} p<0.05$; normal value $=4.0-10 \times 10^{9} / \mathrm{L} ;{ }^{\circ}$ normal value $=<300 \times 10^{6} / \mathrm{L} ;{ }^{\circ 00}$ normal value $=150-400 \times 10^{9} / \mathrm{L} ;{ }^{\square}=$ normal value $<32.0 \mathrm{mg} / \mathrm{mL}$ (according to the European and Dutch criteria) [54] [55]; (i.c.) = intracutaneous tests; "Total IgE in the serum (PRIST)-normal value $\leq 500 \mathrm{IU} / \mathrm{mL}$; "Positive specific IgE in the serum (RAST) $\geq 0.70 \mathrm{U} / \mathrm{mL}$ (= more than class 1); "'Total IgG in the serum (Single radial immuno-diffusion =Mancini technique and ELISA)-normal value $\leq 15.0 \mathrm{~g} / \mathrm{L} ;{ }^{\Delta}$ Normal values: $\operatorname{IgG}_{1}<5.0 \mathrm{~g} / \mathrm{L} ; \operatorname{IgG}_{2}<2.6 \mathrm{~g} / \mathrm{L} ; \operatorname{IgG}_{3}<0.4 \mathrm{~g} / \mathrm{L} ; \mathrm{IgG}_{4}<0.5 \mathrm{~g} / \mathrm{L} ;{ }^{\Delta \Delta}$ Normal values: IgM $\leq 3.8 \mathrm{~g} / \mathrm{L} ;{ }^{\Delta \Delta \Delta}$ Normal values: IgA $\leq 4.0 \mathrm{~g} / \mathrm{L} ;{ }^{\mathbf{\Delta}}=$ measured by flow-cytometry after stimulation with PMA(phorbol 12-myristate 13-acetate) [24]; $\mathbf{\mathbf { A }}_{=}^{=}$measured by ELISA in supernatants of PMA-stimulated peripheral blood mononuclear cells [24]; PBMC = peripheral blood mononuclear cells. 
withdrawn 4 weeks, cromolyn $(n=3)$ and nedocromil sodium $(n=5) 2$ weeks and other treatments 48 hours prior the BPTs. Post-challenge FEV1 decrease by $50 \%$ or more with respect to the pre-challenge values $(\mathrm{n}=3)$ was treated with a single dose of 200 - 400 mcg Salbutamol aerosol. In all study participants a single determination of cytokines released from peripheral blood cells after an "in vitro" stimulation with corresponding allergens was performed (Table 2). In the DYAR patients the BPTs and PBS controls were repeated 2 - 6 weeks later (Figure 1) and supplemented with recording of cytokines released by isolated peripheral blood cells after the "in vitro" stimulation with relevant allergens before and at 1, 12, 24, 36, 48, 56 and 72 hours after the challenge. The local ethical committee approved this study and an informed consent was obtained from all participants.

\subsection{Control Subjects}

The 24 patients demonstrating an IAR, 16 developing LAR, 17 showing DAR and 25 healthy subjects volunteered to participate as control subjects (Tables 1-3).

\subsection{Allergens}

Dialyzed and lyophilized allergen extracts (Allergopharma, Reinbek, Germany) diluted in PBS were used in concentrations of 100 - $500 \mathrm{BU} / \mathrm{mL}$ for skin tests and $1000-3000 \mathrm{BU} / \mathrm{mL}$ for BPTs (Table 3). The concentrations recommended by the manufacturer were $500 \mathrm{BU} / \mathrm{mL}$ for skin tests and $5000 \mathrm{BU} / \mathrm{mL}$ for the BPTs [24].

\subsection{Skin Tests}

Skin prick tests (SPT) with allergenic extracts in concentrations of $500 \mathrm{BU} / \mathrm{mL}$ were evaluated after 20 minutes. If they were negative, intracutaneous tests in concentration of $100 \mathrm{BU} / \mathrm{mL}$ and then $500 \mathrm{BU} / \mathrm{mL}$ were performed and evaluated 20 minutes, 6, 12, 24, 36, 48, 72 and 96 hours after the intradermal injection. A skin wheal (>7.0 $\mathrm{mm}$ in diameter) occurring after 20 minutes was qualified as immediate skin response, skin infiltration appearing between 6 - 12 hours as a late skin response and skin induration observed later than 48 hours was considered a delayed skin response [24].

Table 2. Allergens caused particular types of asthmatic response.

\begin{tabular}{|c|c|c|c|c|c|c|}
\hline Allergen & $\begin{array}{c}\text { Concentration } \\
\text { BU/mL }\end{array}$ & $\begin{array}{l}\text { DYAR } \\
n=23\end{array}$ & $\begin{array}{c}\text { IAR } \\
n=14\end{array}$ & $\begin{array}{c}\text { LAR } \\
n=16\end{array}$ & $\begin{array}{c}\text { DAR } \\
\mathrm{n}=17\end{array}$ & $\begin{array}{l}\text { Healthy subjects } \\
\qquad n=25\end{array}$ \\
\hline Dermatophagoides pteronyss & 1000 & 6 & 9 & 3 & 5 & 0 \\
\hline Dermatophagoides farinae & 1000 & 1 & 1 & 2 & 0 & 0 \\
\hline Animal danders & & & & & & 0 \\
\hline Dog & 3000 & 1 & 2 & 1 & 1 & 0 \\
\hline Cat & 1000 & 1 & 1 & 1 & 2 & 0 \\
\hline Horse & 2000 & 0 & 0 & 1 & 0 & 0 \\
\hline Hamster & 2000 & 1 & 0 & 1 & 0 & 0 \\
\hline Aspergillus fumigatus & 1000 & 1 & 0 & 1 & 1 & 0 \\
\hline Pollen & & & & & & 0 \\
\hline Grass mix I & 1000 & 5 & 4 & 3 & 3 & 0 \\
\hline Grass mix II & 1000 & 2 & 3 & 1 & 0 & 0 \\
\hline Tree mix & 3000 & 1 & 1 & 0 & 1 & 0 \\
\hline Weed mix & 1000 & 1 & 1 & 0 & 1 & 0 \\
\hline Birch & 1000 & 2 & 1 & 1 & 1 & 0 \\
\hline Poplar & 2000 & 1 & 0 & 0 & 1 & 0 \\
\hline Ragweed giant & 1000 & 0 & 1 & 1 & 1 & 0 \\
\hline
\end{tabular}

$\mathrm{BU} / \mathrm{mL}=$ biologic units per $1 \mathrm{~mL}$ Grass pollen mix I = Dactylis glomerata, Lolium perenne, Phleum pratensis, Poa pratensis; Grass pollen mix II = Festuca pratensis, Holcus lanatus, Agrostis alba, Anthoxanthum odoratum; Tree pollen mix = Betula pendula, Corylus avellana, Juniperus communis, Salix alba; Weed pollen mix = Artemisia vulgaris, Plantago lanceolata, Rumex acetosa, Taraxacum officinale. 
Table 3. Single determination of cytokine concentrations in the supernatants of peripheral blood cells stimulated "in vitro" by relevant allergens $(\mathrm{pg} / \mathrm{mL})$.

\begin{tabular}{|c|c|c|c|c|c|}
\hline & \multicolumn{5}{|c|}{ Control subjects } \\
\hline & \multirow{2}{*}{$\begin{array}{c}\text { Patients } \\
\text { DYAR } n=23\end{array}$} & \multicolumn{3}{|c|}{ Patients with asthma } & \multirow{2}{*}{$\begin{array}{l}\text { Healthy subjects } \\
\qquad \mathrm{n}=25\end{array}$} \\
\hline & & IAR $n=24$ & $\mathrm{LAR} n=16$ & DAR $n=17$ & \\
\hline IL-1 $\beta$ & $4.0 \pm 1.1$ & $5.5 \pm 0.8$ & $4.7 \pm 0.3$ & $6.0 \pm 0.9$ & $3.7 \pm 0.6$ \\
\hline IL-2 & $6.9 \pm 0.4^{*}$ & $<3.0$ & $3.1 \pm 0.1$ & $<3.0$ & $<3.0$ \\
\hline IL-3 & $<31.2$ & $37.4 \pm 0.9^{*}$ & $36.2 \pm 2.3^{*}$ & $39.1 \pm 0.7^{*}$ & $<31.2$ \\
\hline IL-4 & $3.7 \pm 1.0$ & $24.6 \pm 2.4^{*}$ & $9.1 \pm 1.5^{+}$ & $12.3 \pm 1.0^{*}$ & $3.2 \pm 0.5$ \\
\hline IL-5 & $4.8 \pm 0.7$ & $11.0 \pm 3.0^{*}$ & $13.5 \pm 2.8^{*}$ & $12.6 \pm 2.2^{*}$ & $<3.0$ \\
\hline IL-6 & $5.0 \pm 0.8$ & $11.6 \pm 1.8^{*}$ & $16.3 \pm 2.0^{*}$ & $18.8 \pm 3.3^{*}$ & $<4.0$ \\
\hline IL-7 & $4.8 \pm 0.5$ & $5.2 \pm 0.3$ & $7.9 \pm 1.1^{+}$ & $5.5 \pm 0.4$ & $4.0 \pm 0.5$ \\
\hline IL-8 & $10.2 \pm 2.1$ & $9.5 \pm 2.5$ & $14.1 \pm 1.9^{*}$ & $10.4 \pm 3.2$ & $8.9 \pm 2.4$ \\
\hline IL-10 & $7.7 \pm 2.2$ & $15.8 \pm 1.4^{*}$ & $11.3 \pm 2.0$ & $12.9 \pm 1.6$ & $8.0 \pm 1.3$ \\
\hline IL-12p40 & $7.8 \pm 0.6$ & $19.3 \pm 3.5^{*}$ & $16.7 \pm 2.9^{*}$ & $15.8 \pm 1.4^{+}$ & $8.5 \pm 2.2$ \\
\hline IL-12p70 & $15.3 \pm 1.6$ & $17.8 \pm 3.1^{+}$ & $15.0 \pm 1.0$ & $14.8 \pm 2.4$ & $12.7 \pm 3.1$ \\
\hline IL-13 & $<3.0$ & $5.0 \pm 0.4$ & $8.8 \pm 2.0^{+}$ & $6.7 \pm 1.2$ & $<3.0$ \\
\hline IL-16 & $59.6 \pm 2.4$ & $55.4 \pm 3.8$ & $59.2 \pm 2.9$ & $54.0 \pm 2.5$ & $56.8 \pm 3.1$ \\
\hline IL-17 & $12.3 \pm 3.0^{*}$ & $7.4 \pm 2.0$ & $6.8 \pm 1.7$ & $8.2 \pm 1.3$ & $6.6 \pm 1.4$ \\
\hline IL-18 & $20.1 \pm 1.3$ & $19.6 \pm 3.8$ & $20.5 \pm 4.1$ & $20.7 \pm 3.0$ & $18.1 \pm 1.9$ \\
\hline IFN- $\gamma$ & $397 \pm 101^{*}$ & $176 \pm 29$ & $210 \pm 35$ & $235 \pm 42$ & $211 \pm 25$ \\
\hline GM-CSF & $12.1 \pm 0.7^{+}$ & $6.7 \pm 1.1$ & $7.0 \pm 0.4$ & $7.3 \pm 0.7$ & $8.2 \pm 0.5$ \\
\hline G-CSF & $8.1 \pm 0.6^{*}$ & $3.9 \pm 0.6$ & $4.2 \pm 0.2$ & $4.1 \pm 0.5$ & $3.8 \pm 0.9$ \\
\hline TNF- $\alpha$ & $12.4 \pm 1.1$ & $10.1 \pm 2.7$ & $11.3 \pm 3.0$ & $10.6 \pm 2.5$ & $9.2 \pm 1.6$ \\
\hline TGF- $\beta$ & $16.1 \pm 1.3$ & $21.3 \pm 4.1$ & $24.8 \pm 3.7^{+}$ & $23.0 \pm 3.5^{+}$ & $15.0 \pm 1.7$ \\
\hline
\end{tabular}

DYAR = delayed asthmatic response; IAR = immediate/early asthmatic response; LAR = late asthmatic response; DAR = dual late asthmatic response; Values = mean \pm SEM; Statistical significance as compared with healthy subject values: ${ }^{+}=\mathrm{p} \leq 0.05,{ }^{*}=\mathrm{p}<0.05 ;{ }^{\Delta}=$ blood cells of the healthy subjects were stimulated with: D. pteronyssinus, Grass mix I, Grass, mix II dog and cat allergens.

\subsection{Bronchial Provocation Tests (BPT)}

The BPTs were performed by means of spirometry (Spirograph D-75 Lode, Groningen, The Netherlands) recording the FVC and FEV1 values. The aerosolized allergen extracts and PBS were inhaled using the Wiebadener Doppel-Inhalator at an airflow of $10 \mathrm{~L} / \mathrm{min}$. The nebulizer output was $0.12-0.14 \mathrm{~mL} / \mathrm{min}$ and the aerosol particles were of a median mass diameter of 2.8 - 3.6 $\mu$. The BPTs, being a modification of the European standard [27], were performed by the following schedule: 1 ) initial (baseline) values recorded at 0,5 and 10 minutes; 2) PBS control values recorded at 0,5 and 10 minutes after a 10-minute PBS inhalation; 3) inhalation of allergen aerosol for $2 \times 5$ minutes, with inserted spirometric value measurement, followed by the recording of the FEV1 and FVC values at $0,5,10,20,30,45,60,90$ and 120 minutes and the every hour up to $12^{\text {th }}$ hour and every second hour during the $22^{\text {nd }}$ and $38^{\text {th }}$, the $46^{\text {th }}$ and $62^{\text {nd }}$ and $72^{\text {nd }}$ hour interval [23]-[26]. The PBS control challenge was performed according the same schedule as that applied to the BPTs with allergens. A 5-day interval has always been inserted between the consecutive tests [23]-[26].

\subsection{Determination of Cytokines}

Samples of $6 \mathrm{~mL}$ heparinized peripheral blood were centrifuged at $1800 \times \mathrm{g}$ for 5 minutes at $4^{\circ} \mathrm{C}$ and plasma aliquots were stored at $-70^{\circ} \mathrm{C}$. The unseparated blood cells were washed in RPMI 1640 (Sigma-Aldrich, St. 
Louis, USA), centrifuged at $2000 \times \mathrm{g}$ for 10 minutes at $4^{\circ} \mathrm{C}$ and re-suspended in RPMI 1640 with penicillin (100 $\mathrm{IU} / \mathrm{mL})$, streptomycin $(100 \mu \mathrm{g} / \mathrm{mL})$ and L-glutamine $(2 \mathrm{mmol} / \mathrm{L})$ at a concentration of $5 \times 10^{6}$ leukocytes $/ \mathrm{mL}$. The cell viability was confirmed by trypan blue dye exclusion. The cell suspension was divided into 2 equal portions. The first portion stimulated with the allergen, identical to that causing the DYAR, in a concentration of $50 \mathrm{BU} / \mathrm{mL}$ and the second non-stimulated control portion were cultured for 24 hours at $37^{\circ} \mathrm{C}$ under $5 \% \mathrm{CO}_{2}$ in a humidified incubator. The supernatants were collected by centrifugation at $2000 \times \mathrm{g}$ for 15 minutes and processed within 1 hour. The cytokines in the supernatants were determined by immunoassay (ELISA) kits, following the manufacturers' recommendations. All measurements were performed in duplicate. The detection limits in $\mathrm{pg} / \mathrm{mL}$ are reported in parenthesis. The cytokines Il-1 $\beta$ (1.0), IL-2 (<3.0), IL-3 (31.2), IL-5 (3.0), IL-6 (4.0), IL-7 (0.1), IL-10 (<3.0),IL-13 (<3.0), IL-16 (31.2), GM-CSF (<3.0), G-CSF (0.8), TNF- $\alpha$ (6.0), TGF- $\beta$ (6.0) were measured using the R \& D System (Minneapolis/MN, USA) kits, IL-4 (0.6), IL-8 (1.3), IL-12p70 (2.1), IL-18 (9.2) and IFN- $\gamma$ (1.0) by Bender MedSystems (Wien, Austria), IL-17 (<5.0) by BioSource International Inc (Camarillo, USA) and IL-12p40 (3.9) by Becton Dickinson (San Jose, USA) kits. The inter-assay as well as intra-assay coefficients of variations for these kits were less than $10 \%$. The blood cells of the healthy controls were stimulated separately with D. pteronyssinus, Grass mix I + II, dog and cat allergens in concentrations of 50 $\mathrm{BU} / \mathrm{mL}$ and the total mean cytokine values were then calculated.

\subsection{Statistical Analysis}

The initial and repeated DYAR and PBS controls were statistically analyzed by means of fitting polynomials to the mean curves over time; eight times points within 120 minutes and twenty-five times points up to 72 hours after the challenge. The hypotheses were tested by the generalized multivariate analysis of the variance model (MANOVA) [28].

The post-challenge cytokine values during the repeated DYAR and PBS controls in individual patients were compared with their pre-challenge values and evaluated by Wilcoxon matched-pair signed rank test. The mean post-challenge values of particular cytokines during the repeated DYARs were compared with the corresponding mean PBS values and evaluated by Mann-Whitney $U$ test. A $p$ value $<0.05$ was considered to be statistically significant.

\section{Results}

The initial DYARs as well as the repeated DYARs, (Figure 1) were significantly positive both in the comparison of the post-challenge with the pre-challenge FEV1 values $(p<0.001, p<0.001$ respectively) and as compared with the PBS control values ( $p<0.001, p<0.001$, respectively). No significant differences were found between the initial and the repeated DYAR $(p>0.2)$ nor between the DYAR appearance and the individual allergens $(p>0.1)$ (Table 2).

The DYAR was associated with decreased bronchial threshold in 73\%, immediate skin response in $35 \%$ and delayed skin response in 57\% (Table 1). The DYAR was accompanied by increased blood leukocyte, neutrophil, monocyte and lymphocyte counts, changed Th1/Th2 cell ratio in peripheral blood in favour of Th1 cells $(p<$ $0.01)$ and increased intracellular concentration of IFN- $\gamma(p<0.01)$ but not of IL-4 ( $p>0.05)$ (Table 4). The DYAR patients, as compared with healthy subjects, demonstrated increased concentrations of IL-2, IL-17, IFN- $\gamma$ and G-CSF $(p<0.05)$ released by the allergen-stimulated blood cells "in vitro". The cytokine profiles of the DYAR patients differed also from those measured in the control patients demonstrating IAR, LAR and DAR (Table 3). The DYAR was associated with following post-challenge cytokine concentrations released by peripheral blood cells stimulated "in vitro" with relevant allergens, as compared both with their pre-challenge and with the corresponding PBS control values: (I). A slight increase $(p \leq 0.05)$ in IL-13, G-CSF and a significant increase $(p<0.05)$ in IL-1 $\beta$, IL-2, IL-8, IL-12p70, IL-18, IFN- $\gamma$, TNF- $\alpha$; (II). A slight decrease $(p \leq 0.05)$ in IL-5, GM-CSF, and a significant decrease $(p<0.05)$ in IL-4, IL-6, IL-17 (Table 5, Figures 2(a) and (b)). Most of these changes occurred 24 - 56 hours after the allergen challenge, which is the time of the maximal DYAR performance. No significant changes in the cytokine concentrations $(p>0.1)$ were found in supernatants during the PBS controls.

\section{Control Subjects}

The IAR, LAR or DAR control patients, as compared with healthy control subjects, demonstrated significant 
Table 4. Differential count in peripheral blood and some cytokines in the serum during the DYAR and PBS.

\begin{tabular}{|c|c|c|c|c|c|c|c|c|c|}
\hline \multirow[b]{2}{*}{$\begin{array}{c}\text { Patients } \\
\mathrm{n}=23\end{array}$} & \multirow[b]{2}{*}{$\begin{array}{l}\text { Before the } \\
\text { challenge }\end{array}$} & \multicolumn{8}{|c|}{ After the challenge (hrs) } \\
\hline & & 1 & 6 & 12 & 24 & 36 & 48 & 56 & 72 \\
\hline \multicolumn{10}{|c|}{ Leukocytes ${ }^{\square}$} \\
\hline DYAR & $8.9 \pm 0.5$ & $10.1 \pm 0.8$ & $10.5 \pm 0.4$ & $11.9 \pm 0.9^{+}$ & $12.7 \pm 0.5^{*}$ & $13.5 \pm 0.6^{*}$ & $12.9 \pm 0.7^{*}$ & $11.6 \pm 0.3^{+}$ & $10.0 \pm 0.5$ \\
\hline PBS & $8.3 \pm 0.4$ & $7.9 \pm 0.7$ & $7.8 \pm 1.0$ & $8.2 \pm 0.9$ & $7.4 \pm 0.5$ & $8.4 \pm 1.0$ & $8.0 \pm 0.6$ & $7.9 \pm 0.5$ & $8.1 \pm 0.4$ \\
\hline \multicolumn{10}{|c|}{ Eosinophils $^{\square \square}$} \\
\hline DYAR & $293 \pm 27$ & $332 \pm 35$ & $318 \pm 24$ & $343 \pm 30$ & $281 \pm 29$ & $272 \pm 27$ & $301 \pm 30$ & $247 \pm 25$ & $288 \pm 34$ \\
\hline PBS & $285 \pm 21$ & $309 \pm 45$ & $293 \pm 26$ & $268 \pm 22$ & $311 \pm 47$ & $256 \pm 22$ & $295 \pm 29$ & $287 \pm 34$ & $276 \pm 23$ \\
\hline \multicolumn{10}{|c|}{ Neutrophils ${ }^{\square \square}$} \\
\hline DYAR & $6.4 \pm 0.3$ & $6.7 \pm 0.9$ & $6.6 \pm 0.5$ & $10.1 \pm 0.8^{*}$ & $10.5 \pm 0.7^{*}$ & $9.4 \pm 1.1^{*}$ & $8.7 \pm 0.4^{*}$ & $7.1 \pm 0.3$ & $6.2 \pm 0.6$ \\
\hline PBS & $5.9 \pm 1.1$ & $6.0 \pm 0.8$ & $6.9 \pm 0.4$ & $6.1 \pm 0.5$ & $6.5 \pm 0.9$ & $7.1 \pm 0.4$ & $6.8 \pm 0.7$ & $6.2 \pm 0.5$ & $6.4 \pm 0.3$ \\
\hline \multicolumn{10}{|c|}{ Thrombocytes $^{\circ}$} \\
\hline DYAR & $343 \pm 41$ & $320 \pm 25$ & $329 \pm 23$ & $347 \pm 40$ & $368 \pm 34$ & $415 \pm 53$ & $386 \pm 31$ & $319 \pm 27$ & $333 \pm 29$ \\
\hline PBS & $298 \pm 17$ & $336 \pm 37$ & $323 \pm 25$ & $299 \pm 16$ & $354 \pm 27$ & $318 \pm 21$ & $349 \pm 38$ & $301 \pm 20$ & $285 \pm 26$ \\
\hline \multicolumn{10}{|c|}{ Differential count ${ }^{\circ}$} \\
\hline \multicolumn{10}{|c|}{ Eosinophils } \\
\hline DYAR & 0.41 & 0.52 & 0.40 & 0.58 & 0.39 & 0.53 & 0.36 & 0.38 & 0.29 \\
\hline PBS & 0.34 & 0.37 & 0.39 & 0.34 & 0.26 & 0.43 & 0.25 & 0.26 & 0.19 \\
\hline \multicolumn{10}{|c|}{ Neutrophils } \\
\hline DYAR & 6.4 & 6.8 & 6.5 & $8.5^{*}$ & $9.4^{*}$ & $9.8^{*}$ & $9.1^{*}$ & $8.9^{*}$ & 7.0 \\
\hline PBS & 6.0 & 6.3 & 6.7 & 5.5 & 6.6 & 6.9 & 5.8 & 7.1 & 5.9 \\
\hline \multicolumn{10}{|l|}{ Basophils } \\
\hline DYAR & 0.06 & 0.09 & 0.10 & 0.05 & 0.10 & 0.05 & 0.09 & 0.06 & 0.15 \\
\hline PBS & 0.07 & 0.03 & 0.05 & 0.10 & 0.08 & 0.09 & 0.04 & 0.09 & 0.05 \\
\hline \multicolumn{10}{|c|}{ Monocytes } \\
\hline DYAR & 0.55 & 0.6 & 1.0 & $1.9^{*}$ & $1.8^{*}$ & $1.7^{*}$ & $1.1^{+}$ & 0.5 & 0.6 \\
\hline PBS & 0.35 & 0.2 & 0.7 & 0.6 & 0.5 & 0.35 & 0.45 & 0.25 & 0.5 \\
\hline \multicolumn{10}{|c|}{ Lymphocytes } \\
\hline DYAR & 2.8 & 3.1 & $3.8^{+}$ & 3.5 & $4.7^{*}$ & $4.8^{*}$ & $3.9^{+}$ & 2.5 & 2.4 \\
\hline PBS & 2.4 & 2.2 & 3.4 & 3.7 & 2.9 & 3.0 & 2.6 & 2.3 & 2.5 \\
\hline \multicolumn{10}{|c|}{$\operatorname{Th}_{1} / \operatorname{Th}_{2}$ ratio $(\%)^{\Delta}$} \\
\hline DYAR & $8.0 \pm 1.5$ & $8.6 \pm 2.1$ & $9.9 \pm 3.0^{+}$ & $14.7 \pm 4.0^{*}$ & $16.2 \pm 3.5^{*}$ & $17.1 \pm 3.0^{*}$ & $16.4 \pm 3.4^{*}$ & $15.6 \pm 4.5^{*}$ & $9.1 \pm 2.2$ \\
\hline PBS & $7.3 \pm 3.1$ & $8.2 \pm 3.0$ & $9.4 \pm 2.1$ & $7.5 \pm 3.4$ & $8.8 \pm 3.7$ & $9.0 \pm 4.1$ & $9.3 \pm 3.1$ & $8.9 \pm 4.2$ & $7.6 \pm 2.6$ \\
\hline \multicolumn{10}{|c|}{ IFN- $\gamma(\mathrm{pg} / \mathrm{mL})$} \\
\hline DYAR & $2.4 \pm 0.5$ & $2.7 \pm 1.0$ & $4.9 \pm 0.4^{+}$ & $9.2 \pm 1.7^{*}$ & $9.0 \pm 3.1^{*}$ & $8.8 \pm 3.2^{*}$ & $7.1 \pm 1.3^{*}$ & $3.1 \pm 1.0$ & $2.9 \pm 0.3$ \\
\hline PBS & $2.1 \pm 0.2$ & $1.9 \pm 0.8$ & $2.2 \pm 0.6$ & $1.9 \pm 0.8$ & $2.4 \pm 0.5$ & $2.2 \pm 1.0$ & $2.5 \pm 1.0$ & $2.4 \pm 0.5$ & $2.3 \pm 0.2$ \\
\hline \multicolumn{10}{|c|}{ IL-4 (pg/mL) } \\
\hline DYAR & $3.0 \pm 0.1$ & $3.2 \pm 0.1$ & $3.5 \pm 0.4$ & $3.7 \pm 0.6$ & $3.2 \pm 0.4$ & $3.4 \pm 0.3$ & $3.5 \pm 0.5$ & $3.3 \pm 0.5$ & $3.5 \pm 0.4$ \\
\hline PBS & $3.1 \pm 0.2$ & $3.0 \pm 0.2$ & $3.3 \pm 0.1$ & $3.4 \pm 0.5$ & $3.5 \pm 0.2$ & $3.0 \pm 0.2$ & $3.4 \pm 0.7$ & $3.5 \pm 0.6$ & $3.2 \pm 0.1$ \\
\hline
\end{tabular}

DYAR = Delayed asthmatic response; PBS=Phosphate buffered saline (control); Values = mean \pm SEM; Statistical significance as compared with healthy subject values: ${ }^{+}=\mathrm{p} \leq 0.05,{ }^{*}=\mathrm{p}<0.05$, Automated counts: ${ }^{\square}=$ normal value $4.0-10.0 \times 10^{9} / \mathrm{L} ;{ }^{\square}=$ normal value $<300 \times 10^{6} / \mathrm{L} ;{ }^{\square \square}=$ normal value $2.0-7.2 \times 10^{9} / \mathrm{L} ;{ }^{\circ}=$ normal value $150-400 \times 10^{9} / \mathrm{L}$; Manual counts: ${ }^{\circ}=$ normal values $\left(\times 10^{9} / \mathrm{L}\right): \mathrm{E}<0.50 ; \mathrm{N}=2.0-7.2 ; \mathrm{B}<0.2 ; \mathrm{M}=0.2-1.0$; $\mathrm{L}=1.0-4.0{ }^{\Delta}=$ measured by flow-cytometry after stimulation with PMA (Phorbol 12-myristate 12-acetate). 
Table 5. Cytokine released by peripheral blood cells (PMN and PBMC) stimulated “in vitro" with relevant allergens (means \pm SEM) during the DYAR and PBS controls.

\begin{tabular}{|c|c|c|c|c|c|c|c|c|}
\hline \multirow[b]{2}{*}{ Patients $n=23$} & \multirow[b]{2}{*}{$\begin{array}{l}\text { Before the } \\
\text { challenge }\end{array}$} & \multicolumn{7}{|c|}{ After the challenge (hrs) } \\
\hline & & 1 & 12 & 24 & 36 & 48 & 56 & 72 \\
\hline \multicolumn{9}{|l|}{$\mathrm{IL-1 \beta}(\mathrm{pg} / \mathrm{mL})$} \\
\hline DYAR & $4.1 \pm 1.1$ & $5.0 \pm 0.7$ & $6.8 \pm 1.5^{*}$ & $7.0 \pm 0.6^{*}$ & $6.9 \pm 0.5^{+}$ & $5.0 \pm 0.2$ & $5.1 \pm 1.0$ & $4.6 \pm 0.7$ \\
\hline PBS & $2.9 \pm 0.8$ & $4.1 \pm 0.8$ & $4.2 \pm 0.3$ & $3.9 \pm 0.5$ & $3.4 \pm 1.0$ & $3.0 \pm 0.5$ & $3.5 \pm 0.4$ & $3.3 \pm 0.2$ \\
\hline \multicolumn{9}{|l|}{ IL-2 (pg/mL) } \\
\hline DYAR & $6.9 \pm 0.4$ & $5.2 \pm 0.1$ & $9.6 \pm 1.0^{*}$ & $11.3 \pm 0.4^{*}$ & $11.8 \pm 1.7^{*}$ & $10.4 \pm 0.6^{*}$ & $8.9 \pm 0.2^{*}$ & $<3.0$ \\
\hline PBS & $4.8 \pm 0.8$ & $5.0 \pm 0.9$ & $7.1 \pm 0.4$ & $6.0 \pm 0.3$ & $4.8 \pm 0.6$ & $5.3 \pm 0.2$ & $4.9 \pm 0.4$ & $4.5 \pm 0.4$ \\
\hline \multicolumn{9}{|l|}{ IL-3 (pg/mL) } \\
\hline DYAR & $<31.2$ & $<31.2$ & $<31.2$ & $32.1 \pm 0.4$ & $<31.2$ & $<31.2$ & $<31.2$ & $<31.2$ \\
\hline PBS & $<31.2$ & $<31.2$ & $<31.2$ & $<31.2$ & $<31.2$ & $<31.2$ & $<31.2$ & $<31.2$ \\
\hline \multicolumn{9}{|l|}{ IL-4 (pg/mL) } \\
\hline DYAR & $3.7 \pm 1.0$ & $5.0 \pm 0.7$ & $4.1 \pm 0.9$ & $2.0 \pm 0.3$ & $<0.6^{*}$ & $<0.6^{*}$ & $<0.6^{*}$ & $3.3 \pm 0.2$ \\
\hline PBS & $2.9 \pm 0.3$ & $2.7 \pm 1.0$ & $3.6 \pm 0.8$ & $3.4 \pm 0.5$ & $3.7 \pm 0.6$ & $2.2 \pm 0.3$ & $2.6 \pm 0.8$ & $2.5 \pm 1.0$ \\
\hline \multicolumn{9}{|l|}{ IL-5 (pg/mL) } \\
\hline DYAR & $6.9 \pm 1.0$ & $6.7 \pm 0.8$ & $6.5 \pm 1.3$ & $5.0 \pm 0.4^{+}$ & $5.1 \pm 0.7^{+}$ & $6.3 \pm 1.1$ & $7.0 \pm 1.0$ & $6.4 \pm 0.9$ \\
\hline PBS & $6.5 \pm 0.3$ & $7.5 \pm 1.4$ & $7.0 \pm 0.7$ & $7.0 \pm 1.1$ & $7.2 \pm 0.6$ & $7.1 \pm 0.4$ & $6.3 \pm 0.5$ & $6.6 \pm 1.0$ \\
\hline \multicolumn{9}{|l|}{ IL-6 (pg/mL) } \\
\hline DYAR & $5.0 \pm 0.8$ & $5.8 \pm 0.6$ & $8.1 \pm 1.1^{*}$ & $4.6 \pm 0.4$ & $<4.0^{*}$ & $<4.0^{*}$ & $4.5 \pm 0.4$ & $5.0 \pm 0.5$ \\
\hline PBS & $5.1 \pm 0.5$ & $5.1 \pm 1.0$ & $5.2 \pm 0.6$ & $5.9 \pm 1.3$ & $5.0 \pm 1.0$ & $5.4 \pm 0.3$ & $4.7 \pm 0.5$ & $4.5 \pm 0.4$ \\
\hline \multicolumn{9}{|l|}{ IL-7 (pg/mL) } \\
\hline DYAR & $4.8 \pm 0.5$ & $5.7 \pm 1.0$ & $6.2 \pm 0.8$ & $6.5 \pm 0.4$ & $5.9 \pm 1.0$ & $6.6 \pm 0.7$ & $6.3 \pm 0.4$ & $5.8 \pm 0.3$ \\
\hline PBS & $4.4 \pm 0.4$ & $4.9 \pm 0.7$ & $5.5 \pm 0.9$ & $4.8 \pm 0.9$ & $6.0 \pm 1.0$ & $6.3 \pm 0.8$ & $4.6 \pm 0.5$ & $5.3 \pm 0.6$ \\
\hline \multicolumn{9}{|l|}{ IL-8 (pg/mL) } \\
\hline DYAR & $11.2 \pm 2.1$ & $13.6 \pm 1.8$ & $15.5 \pm 1.0^{+}$ & $16.9 \pm 1.6^{*}$ & $17.0 \pm 1.4^{*}$ & $15.8 \pm 1.0^{*}$ & $14.9 \pm 1.1^{+}$ & $12.8 \pm 1.1$ \\
\hline PBS & $10.5 \pm 0.4$ & $11.5 \pm 0.6$ & $12.4 \pm 0.5$ & $12.6 \pm 1.0$ & $13.0 \pm 1.0$ & $12.5 \pm 0.7$ & $11.5 \pm 2.0$ & $10.2 \pm 1.0$ \\
\hline \multicolumn{9}{|l|}{ IL-10 (pg/mL) } \\
\hline DYAR & $10.9 \pm 2.2$ & $10.3 \pm 0.5$ & $11.5 \pm 0.8$ & $11.2 \pm 0.3$ & $11.4 \pm 1.3$ & $11.7 \pm 0.5$ & $11.0 \pm 1.0$ & $10.3 \pm 0.4$ \\
\hline PBS & $9.5 \pm 0.6$ & $10.0 \pm 0.3$ & $9.8 \pm 0.6$ & $10.9 \pm 1.1$ & $11.0 \pm 0.7$ & $10.8 \pm 0.6$ & $9.7 \pm 0.3$ & $10.0 \pm 0.7$ \\
\hline \multicolumn{9}{|l|}{ IL-12p40 (pg/mL) } \\
\hline DYAR & $11.7 \pm 0.6$ & $12.3 \pm 1.1$ & $12.6 \pm 2.0$ & $12.6 \pm 0.9$ & $11.5 \pm 1.2$ & $11.9 \pm 0.7$ & $11.6 \pm 1.0$ & $12.2 \pm 0.6$ \\
\hline PBS & $11.0 \pm 1.4$ & $12.0 \pm 2.0$ & $12.2 \pm 1.0$ & $11.7 \pm 0.7$ & $12.1 \pm 2.0$ & $11.5 \pm 0.5$ & $11.9 \pm 0.8$ & $11.4 \pm 1.1$ \\
\hline \multicolumn{9}{|l|}{ IL-12p70 (pg/mL) } \\
\hline DYAR & $15.3 \pm 1.6$ & $15.7 \pm 1.1$ & $19.4 \pm 2.1^{*}$ & $23.5 \pm 2.5^{*}$ & $24.4 \pm 2.6^{*}$ & $20.1 \pm 3.0^{*}$ & $22.3 \pm 1.8^{*}$ & $16.0 \pm 1.0$ \\
\hline PBS & $15.6 \pm 1.4$ & $15.0 \pm 1.0$ & $16.3 \pm 1.2$ & $16.0 \pm 0.6$ & $17.6 \pm 1.4$ & $17.1 \pm 0.8$ & $16.5 \pm 1.0$ & $17.0 \pm 1.4$ \\
\hline
\end{tabular}




\section{Continued}

\begin{tabular}{|c|c|c|c|c|c|c|c|c|}
\hline \multicolumn{9}{|c|}{ IL-13 (pg/mL) } \\
\hline DYAR & $5.7 \pm 0.5$ & $6.1 \pm 0.8$ & $7.2 \pm 0.6^{+}$ & $7.1 \pm 0.4^{+}$ & $6.3 \pm 1.0$ & $6.2 \pm 1.0$ & $5.5 \pm 0.4$ & $5.8 \pm 0.6$ \\
\hline PBS & $5.0 \pm 0.7$ & $5.5 \pm 1.0$ & $7.3 \pm 0.8$ & $7.3 \pm 1.0$ & $6.6 \pm 1.3$ & $6.1 \pm 1.2$ & $5.9 \pm 0.7$ & $5.6 \pm 0.4$ \\
\hline \multicolumn{9}{|c|}{ IL-16 (pg/mL) } \\
\hline DYAR & $59.6 \pm 2.4$ & $66.3 \pm 3.7$ & $68.0 \pm 3.2$ & $65.0 \pm 2.9$ & $58.6 \pm 4.5$ & $61.4 \pm 3.1$ & $58.5 \pm 2.7$ & $57.0 \pm 2.5$ \\
\hline PBS & $60.7 \pm 4.1$ & $56.5 \pm 3.0$ & $57.7 \pm 2.8$ & $60.2 \pm 3.3$ & $59.9 \pm 4.3$ & $55.4 \pm 3.8$ & $61.2 \pm 3.1$ & $58.2 \pm 2.9$ \\
\hline \multicolumn{9}{|c|}{ IL-17 (pg/mL) } \\
\hline DYAR & $12.3 \pm 3.0$ & $11.6 \pm 2.8$ & $7.4 \pm 3.9^{+}$ & $<5.0^{*}$ & $<5.0^{*}$ & $12.6 \pm 1.5$ & $12.9 \pm 2.2$ & $12.4 \pm 2.1$ \\
\hline PBS & $12.0 \pm 2.3$ & $12.5 \pm 3.1$ & $11.4 \pm 2.5$ & $10.9 \pm 2.2$ & $11.7 \pm 2.8$ & $10.0 \pm 2.6$ & $11.5 \pm 2.9$ & $11.2 \pm 1.5$ \\
\hline \multicolumn{9}{|c|}{ IL-18 (pg/mL) } \\
\hline DYAR & $20.1 \pm 1.3$ & $19.6 \pm 1.0$ & $23.4 \pm 0.8$ & $26.7 \pm 1.5^{+}$ & $27.5 \pm 1.2^{*}$ & $28.0 \pm 2.6^{*}$ & $30.1 \pm 4.0^{*}$ & $26.3 \pm 2.7$ \\
\hline PBS & $19.9 \pm 0.6$ & $20.0 \pm 2.1$ & $22.1 \pm 2.0$ & $21.8 \pm 1.2$ & $22.4 \pm 0.9$ & $21.9 \pm 0.6$ & $21.0 \pm 0.4$ & $20.2 \pm 1.1$ \\
\hline \multicolumn{9}{|c|}{ IFN- $\gamma(\mathbf{p g} / \mathbf{m L})$} \\
\hline DYAR & $385 \pm 101$ & $403 \pm 111$ & $538 \pm 107^{+}$ & $591 \pm 122^{*}$ & $593 \pm 120^{*}$ & $513 \pm 94^{*}$ & $489 \pm 106^{*}$ & $414 \pm 108^{+}$ \\
\hline PBS & $381 \pm 97$ & $377 \pm 98$ & $398 \pm 90$ & $345 \pm 116$ & $355 \pm 121$ & $358 \pm 121$ & $383 \pm 95$ & $374 \pm 101$ \\
\hline \multicolumn{9}{|c|}{ GM-CSF (pg/mL) } \\
\hline DYAR & $12.1 \pm 0.7$ & $13.6 \pm 0.5$ & $10.3 \pm 1.0$ & $9.8 \pm 1.2^{+}$ & $9.9 \pm 2.0^{+}$ & $12.0 \pm 0.9$ & $12.5 \pm 1.2$ & $12.6 \pm 0.7$ \\
\hline PBS & $11.4 \pm 0.5$ & $11.9 \pm 0.8$ & $12.5 \pm 1.1$ & $12.9 \pm 1.0$ & $11.8 \pm 1.3$ & $11.6 \pm 0.5$ & $11.8 \pm 1.0$ & $11.2 \pm 0.9$ \\
\hline \multicolumn{9}{|c|}{ G-CSF (pg/mL) } \\
\hline DYAR & $7.1 \pm 0.6$ & $8.4 \pm 1.0$ & $8.9 \pm 0.7^{+}$ & $9.3 \pm 2.2^{+}$ & $9.0 \pm 1.6^{+}$ & $8.6 \pm 0.7$ & $7.5 \pm 1.2$ & $7.8 \pm 1.1$ \\
\hline PBS & $7.8 \pm 0.5$ & $7.0 \pm 0.4$ & $7.9 \pm 0.5$ & $8.6 \pm 1.1$ & $8.0 \pm 0.3$ & $8.2 \pm 1.4$ & $8.5 \pm 0.9$ & $7.7 \pm 0.9$ \\
\hline \multicolumn{9}{|c|}{ TNF- $\alpha(p g / m L)$} \\
\hline DYAR & $12.4 \pm 1.1$ & $14.2 \pm 0.9$ & $17.8 \pm 1.0^{*}$ & $18.5 \pm 0.6^{*}$ & $18.8 \pm 0.4^{*}$ & $19.0 \pm 2.0^{*}$ & $17.7 \pm 1.1^{*}$ & $16.1 \pm 1.0^{+}$ \\
\hline PBS & $13.0 \pm 0.6$ & $13.9 \pm 1.1$ & $14.4 \pm 1.0$ & $15.0 \pm 1.3$ & $14.7 \pm 1.3$ & $14.0 \pm 2.0$ & $15.0 \pm 1.0$ & $14.2 \pm 0.7$ \\
\hline \multicolumn{9}{|c|}{ TGF- $\beta(\mathrm{pg} / \mathrm{mL})$} \\
\hline DYAR & $17.9 \pm 1.3$ & $18.5 \pm 2.2$ & $19.7 \pm 2.0$ & $18.3 \pm 1.0$ & $19.1 \pm 1.4$ & $17.8 \pm 1.5$ & $18.4 \pm 1.0$ & $17.3 \pm 0.5$ \\
\hline PBS & $18.3 \pm 1.0$ & $18.2 \pm 0.6$ & $19.5 \pm 0.4$ & $19.0 \pm 1.0$ & $20.2 \pm 1.3$ & $19.5 \pm 0.7$ & $18.9 \pm 0.6$ & $19.1 \pm 0.4$ \\
\hline
\end{tabular}

DYAR = Delayed asthmatic response; PBS= Phosphate buffered saline (control); Values of cytokines = means \pm SEM; Statistical significance of the cytokine concentrations as compared with their pre-challenge (baseline) values: ${ }^{+}=p \leq 0.05 ;{ }^{*}=p<0.05$.

changes in the (pre-challenge) concentrations of various cytokines in the supernatants of the blood cells stimulated "in vitro" with relevant allergens, differing also from concentrations measured in the DYAR patients (Table 3).

\section{Discussion}

The DYAR differs from IAR, LAR and DAR in clinical course and accompanying features, such as cellular changes and different profiles of eicosanoids, adhesion molecules and cytokines in peripheral blood [1] [3]-[8] [12]-[15] [20] [23]-[26]. These differences suggest involvement of different immunologic mechanisms in the particular asthmatic response types. Cytokines expressing manifold biologic effects, including those on cellular signal transmission, stimulation, inhibition, chemotaxis and cell migration, transmembrane metabolism and 


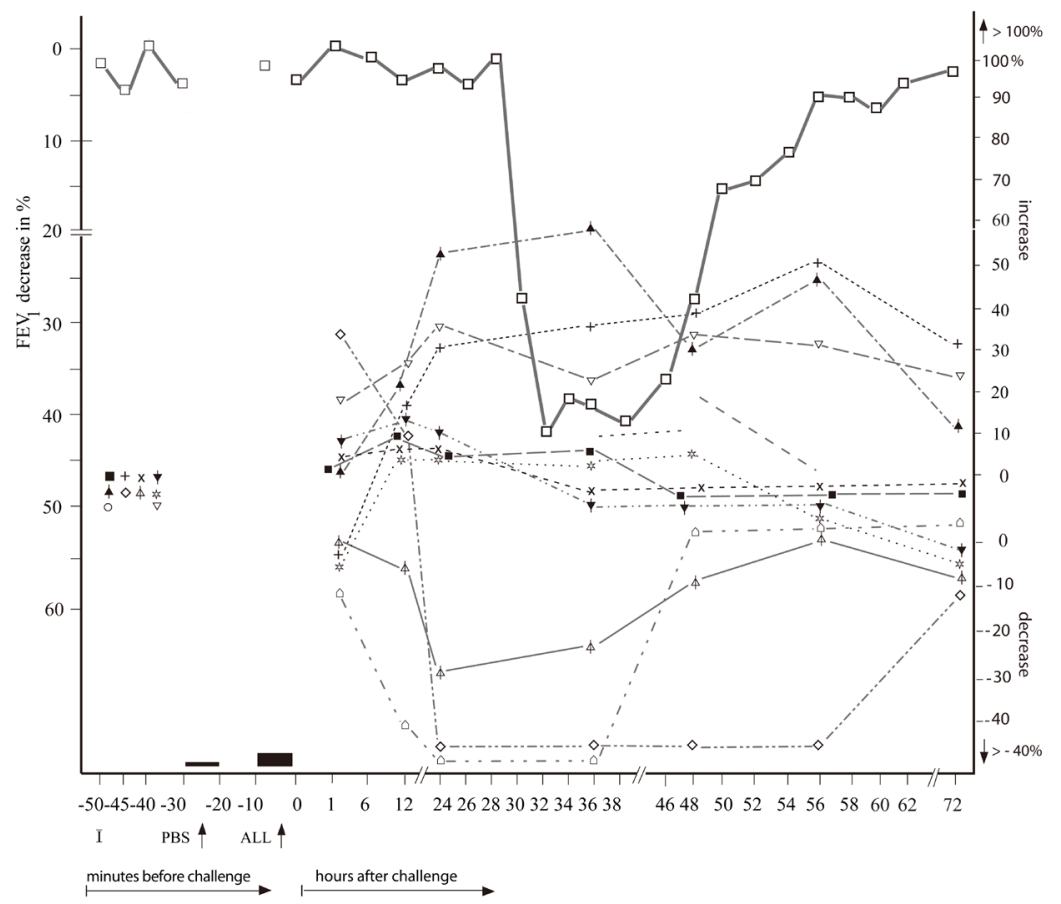

(a)

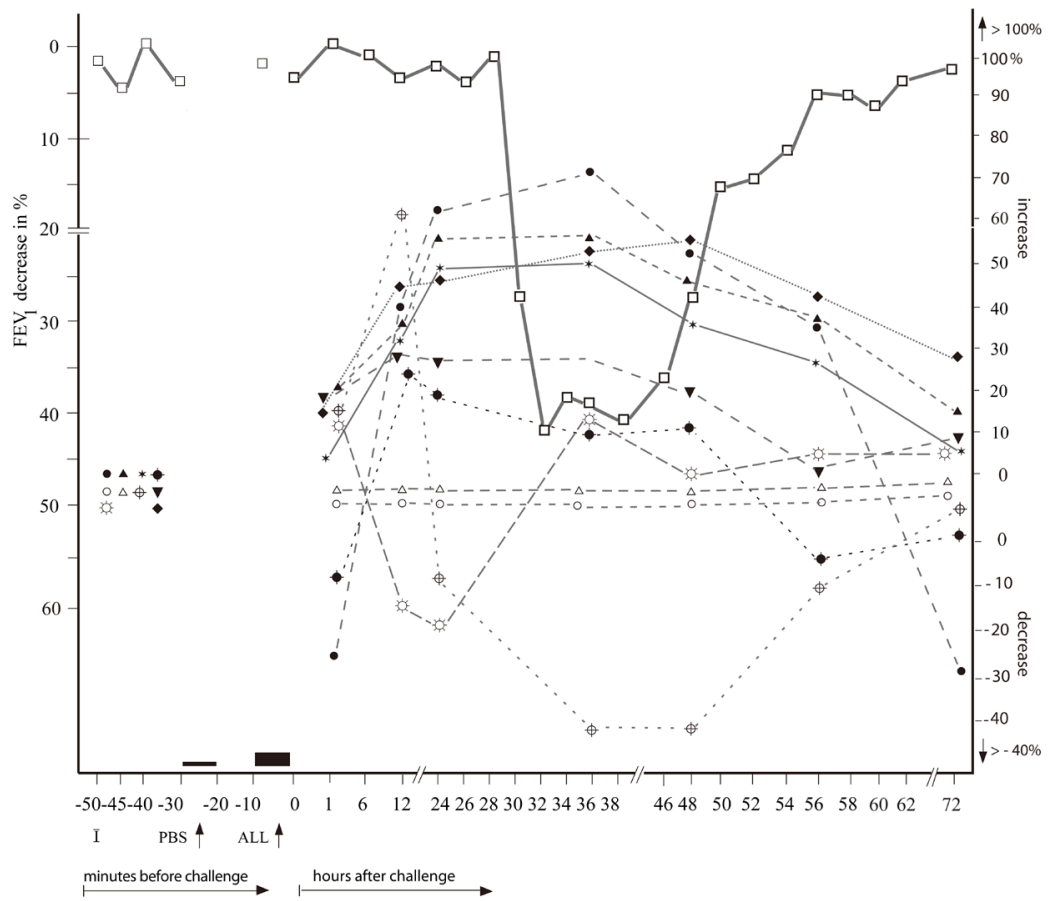

(b)

Figure 2. The repeated delayed asthmatic response to allergen challenge (DYAR) $(\square-\square)$ and the mean percentage changes in the concentrations of particular cytokines released from isolated blood cells stimulated with allergen "in vitro". (a) $\Delta=$

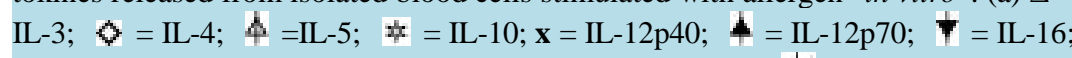
$\triangle=\mathrm{IL}-17 ;+=\mathrm{IL}-18 ; \mathbf{\square}=\mathrm{TGF}-\beta ;$ (b) $\circ=\mathrm{IL}-1 \beta ; \bullet=\mathrm{IL}-2 ; \quad \phi=\mathrm{IL}-6 ; \quad \nabla=\mathrm{IL}-7 ;$ $\boldsymbol{\Delta}=\mathrm{IL}-8 ; \quad=\mathrm{IL}-13 ; \quad$ * $=\mathrm{IFN}-\gamma ; \quad=\mathrm{TNF}-\alpha ;$ Initial (baseline) value; ALL = Allergen challenge; PBS= Phosphate buffered saline. 
maturation of various cell types, represent important parts of the immunologic mechanisms [1] [4] [6] [9] [14] [16] [22] [29] [30]. Their role in the immunologic mechanisms underlying the allergic bronchial asthma has already been investigated from various points of view [4] [6] [8]-[11] [14]-[20] [24] [25] [29]-[52].

In most of these studies, a single measurement of cytokines in bronchoalveolar lavage fluid (BAL), (induced) sputum or in the peripheral blood was performed [9]-[11] [17] [19] [32]-[34] [38] [40] [42] [43] [45]-[49] [51] [52]. Studies dealing with the cytokine determination after the allergen challenge, especially during the IAR, LAR or DAR, are not numerous [5] [6] [14] [15] [18] [20] [30] [31] [39] [41] [50]. The concentration changes of particular cytokines in asthma patients reported in the literature display relatively high variation. This result diversity is probably caused by factors related either to the investigated patient populations, such as different asthma phenotypes, extent of the diagnostic procedures and selection criteria or to the differences in the methods, such as medium studied, technique and timing of the material collection, bronchial provocation technique (inhalational, intra-bronchial, segmental challenge), and the processing of collected material [2] [5]-[8] [14]-[20] [22] [24] [29] [30] [32] [38] [40] [41] [45] [52]. Usually, the concentrations of cytokines released by the circulating or airway-related cells into the peripheral blood by "in vivo" inhaled allergens are lower than the cytokine amounts released by "in vitro" stimulation of the isolated cells with various agents/factors [8] [15] [20]. The "in vitro" stimulation of the isolated blood or the airway-related cells, can be performed with non-specific agents, e.g. PHA , PMA, fMLP, LPS [9] [10] [17] [18] [32] [48], bacterial enterotoxin, other cytokines [6] [34]-[37] [39] [44] [46], or with allergens [6] [9] [33] [34] [38] [40] [42] [43] [45]-[49] [51] [52].

Of the "in vitro" techniques, the stimulation of isolated cells with allergens produces relatively limited cytokine amounts, stimulation with cytokines can release larger cytokine amounts, and the stimulation with non-specific agents generates the largest cytokines amounts [11] [24] [45] [46] [48] [49]. The differences in the released cytokine amounts can be explained by the mode of action of the stimulating agents. The stimulation with the non-specific, but very powerful, stimulating agents results in a direct release of the almost complete intracellular cytokine potential, including even those portions which would not be released upon natural allergen inhalation. In contrast, stimulation with specific allergens results in a selective release of a part of the total intracellular cytokine capacity only. Moreover, the stimulation of the isolated cells with allergen lacks a number of factors and mechanisms involved in the "in vivo" process, such as antigen presentation by the antigen presenting cells, synergic and antagonistic effects of other cells and factors in the bronchial tissue as well as in the peripheral blood, and concomitant effects of other cytokines and chemokines [1] [4] [6] [8] [14] [18] [21] [34].

The measurement of the cytokines released by the allergen-stimulated blood cells "in vitro" is important for the evaluation of the ratio of particular cytokines released by allergen stimulation with respect to their total intracellular capacity released by their stimulation with non-specific agents, and should be interpreted as a model for studying of these processes. However, the cytokine determination in the natural media, such as blood or sputum, related to the allergen inhaled during the bronchial challenge would be more representative for the genuine processes involved in the clinical bronchial asthma. This fact may be supported by our , not yet published, results of higher cytokine concentrations released after the "in vitro" stimulation with PMA than cytokine amounts recorded in plasma after the bronchial allergen challenge as well as by similar findings of other investigators [40] [42] [45] [49]. Unfortunately, the discrepancy between the cytokine amounts released by the "in vivo" inhaled allergen and those released after the "in vitro" stimulation of the isolated BAL or peripheral blood cells with non-specific agents during particular asthmatic response types, has not yet been sufficiently investigated. Moreover, the cytokine profiles in the peripheral blood, BAL fluid or sputum as well as after stimulation of isolated blood cells with various agents, including allergens, in healthy subjects, which would served as reference data, have as yet been insufficiently investigated [9] [29] [32] [47] [49].

In majority of the papers, studying the cytokines in bronchial asthma patients, the single cytokine determination was performed [9] [10] [17] [33] [34] [38] [40] [42] [43] [45]-[49] [51] [52]. However, our data (Table 5) show that the significant changes of the cytokine concentrations were measured till 24 - 56 hours after the allergen challenge. This fact would emphasize the importance of cytokine measuring before and repeatedly after the bronchial allergen challenge, being the only method capable of following the dynamic course of the cytokine changes related to the allergen exposure [3] [5] [14] [15] [18] [20] [23]-[26] [30] [31] [39] [41] [50]. Results of this study, consistent with our previous findings [23]-[26] [53], suggest that immunologic mechanisms underlying the DYAR differ from those involved both in IAR and in LAR. The pre-challenge serum cytokine levels recorded in the DYAR patients [53] as well as those released from the allergen-stimulated blood cells "in vitro" (Table 3) differed from those measured in the IAR, LAR or DAR patients. The "in vitro" allergen-stimulated 
blood cells released increased amount of IL-1 $\beta$, IL-2, IL-8, IL-12p70, IL-13, IL-18, IFN- $\gamma$ and TNF- $\alpha$, while decreased amounts of IL-4, IL-6 and IL-17 (Table 5). These cytokine profiles differed from those recorded in the serum during the DYAR [53]. These differences may probably lie in various, above mentioned, factors and mechanisms participating in the cytokine release "in vivo", but lacking in the "in vitro" processes. An interesting aspect was a prevalence of changes of pro-inflammatory cytokines.

The post-challenge cytokine profiles released by the allergen-stimulated blood cells during the DYAR differed from other investigator findings reporting increased release of IL-1 $\beta$, IL-4, IL-5, IL-6, IL-9, IL-10, IL-13, IL-16, IFN- $\gamma$, or TFN- $\alpha$ by blood mononuclear cells of asthmatics due to the stimulation with inhalant allergens. However, in most of these studies, only a limited cytokine number has been measured and even without any relation to a particular asthmatic response type [11] [33] [34] [38] [45]-[49]. The cytokine profiles reported in this study, together with our previous results [23]-[26] [53], especially with the significant changes in the Th1/Th2 ratio in peripheral blood in favour of Th1 cells, increased intracellular concentrations of IFN- $\gamma$ and IL-2, but not those of IL-4 or IL-5, increased blood leukocyte, neutrophil and monocyte, but not eosinophil, counts, increased plasma levels of of LTB4 and MPO and serum concentration changes of various soluble adhesion molecules would suggest involvement of the cell-mediated hypersensitivity upon participation of Th1 lymphocytes, neutrophils, monocytes, probably also macrophages, NK cells, epithelial and endothelial cells, in the DYAR. Nevertheless, more concurrent investigations should be performed to clarify the immunologic mechanisms underlying the DYAR.

\section{References}

[1] Holgate, S.T., Lemanske, R.F., O’Byrne, P.M., Kukumanu, S. and Busse, W.W. (2009) Asthma Pathogenesis. In: Adkinson, N.F., Bochner, B.S., Busse, W.W., Holgate, S.T., Lemanske, R.F. and Simons, F.E., Eds., Middleton's Allergy, Principles and Practice, 7th Edition, Mosby-Elsevier, Philadelphia, 893-919.

[2] Green, R.H., Brightling, C.E. and Bradding, P. (2007) The Reclassification of Asthma Based on Subphenotypes. Current Opinion in Allergy and Clinical Immunology, 7, 43-50. http://dx.doi.org/10.1097/ACI.0b013e3280118a32

[3] Sheth, K.K. and Lemanske, R.F. (1995) The Early and Late Asthmatic Response to Allergen Challenge. In: Busse, W.W. and Holgate, S.T., Eds., Asthma and Rhinitis, Blackwell Scientific Publication, Oxford, 946-960.

[4] Borish, L. and Rosenwasser, L.J. (2009) Cytokines in Allergic Inflammation. In: Adkinson, N.F., Bochner, B.S., Busse, W.W., Holgate, S.T., Lemanske, R.F. and Simons, F.E., Eds., Middleton's Allergy, Principles and Practice, 7th Edition, Mosby-Elsevier, Philadelphia, 165-179.

[5] Jarjour, N.N., Calhoun, W.J., Kelly, E.A., Gleich, G.J., Schwartz, L.B. and Busse, W.W. (1997) The Immediate and Late Allergic Response to Segmental Bronchopulmonary Provocation in Asthma. American Journal of Respiratory and Critical Care Medicine, 155, 1515-1521. http://dx.doi.org/10.1164/ajrccm.155.5.9154851

[6] Leonard, C., Tormey, V., Burke, C. and Poulter, L.W. (1997) Allergen-Induced Cytokine Production in Atopic Disease and Its Relationship to Disease Severity. American Journal of Respiratory Cell and Molecular Biology, 17, 368-375. http://dx.doi.org/10.1165/ajrcmb.17.3.2797

[7] Pelikan, Z., Pelikan-Filipek, M., Kruis, M. and Berger, M.P.F. (1986) The Immediate Asthmatic Response to Allergen Challenge. Annals of Allergy, 56, 252-260.

[8] Pelikan, Z. and Pelikan-Filipek, M. (1986) The Late Asthmatic Response to Allergen Challenge-Part I and II. Annals of Allergy, 56, 414-420,421-435.

[9] Wong, C.K., Ho, C.Y., Ko, F.W.S., Chan, C.H.S., Ho, A.S.S., Hui, D.S.C. and Lam, C.W.K. (2001) Proinflammatory Cytokines (IL-17, IL-6, IL-18 and IL-12) and Th Cytokines (IFN- $\gamma$, IL-4, IL-10 and IL-13) in Patients with Allergic Asthma. Clinical \& Experimental Immunology, 125, 177-183. http://dx.doi.org/10.1046/j.1365-2249.2001.01602.x

[10] Shirai, T., Suzuki, K., Inui, N., Suda, T., Chida, K. and Nakamura, H. (2003) Th1/Th2 Profile in Peripheral Blood in Atopic Cough and Atopic Asthma. Clinical \& Experimental Allergy, 33, 84-89. http://dx.doi.org/10.1046/j.1365-2222.2003.01578.x

[11] Smart, J.M. and Kemp, A.S. (2002) Increased Th 1 and Th 2 Allergen-Induced Cytokine Responses in Children with Atopic Diseases. Clinical \& Experimental Allergy, 32, 796-802. http://dx.doi.org/10.1046/j.1365-2222.2002.01391.x

[12] Pelikan, Z., Pelikan-Filipek, M. and van Oers, J. (2001) The Early Asthmatic Response to Allergen Challenge (EAR) and Its Pharmacologic Modulation. Allergy, 56, 24.

[13] Pelikan, Z. (2001) The Effects of Various Drugs on the Late Asthmatic Response (LAR) to the Bronchial Challenge with Allergens [BPT]. Allergy, 56, 25.

[14] Yoshida, M., Watson, R.M., Rerecich, T. and O’Byrne, P.M. (2005) Different Profiles of T-Cell IFN- $\gamma$ and IL-12 in 
Allergen-Induced Early and Dual Responders with Asthma. Journal of Allergy and Clinical Immunology, 115, 10041009. http://dx.doi.org/10.1016/j.jaci.2005.02.003

[15] Gratziou, C., Carroll, M., Montefort, S., Teran, L., Howarth, P.H. and Holgate, S.T. (1996) Inflammatory and T-Cell Profile of Asthmatic Airways 6 Hours after Local Allergen Provocation. American Journal of Respiratory and Critical Care Medicine, 153, 515-520. http://dx.doi.org/10.1164/ajrccm.153.2.8564090

[16] Bodey, K.J., Semper, A.E., Redington, A.E., Madden, J., Teran, L.M., Holgate, S.T. and Frew, A.J. (1999) Cytokine Profiles of BAL T Cells and T-Cell Clones Obtained from Human Asthmatic Airways after Local Allergen Challenge. Allergy, 54, 1083-1093. http://dx.doi.org/10.1034/j.1398-9995.1999.00889.x

[17] Majori, M., Corradi, M., Caminati, A., Cacciani, G., Bertacco, S. and Pesci, A. (1999) Predominant Th 1 Cytokine Pattern in Peripheral Blood from Subjects with Chronic Pulmonary Obstructive Disease. Journal of Allergy and Clinical Immunology, 103, 458-462. http://dx.doi.org/10.1016/S0091-6749(99)70471-9

[18] Liu, L., Jarjour, N.N., Busse, W.W. and Kelly, E.A.B. (2004) Enhanced Generation of Helper T Type 1 and 2 Chemokines in Allergen-Induced Asthma. American Journal of Respiratory and Critical Care Medicine, 169, 1118-1124. http://dx.doi.org/10.1164/rccm.200312-16590C

[19] Krug, N., Madden, J., Redington, A.E., Lackie, P., Djukanovic, R., Schauer, U., Holgate, S.T., Frew, A.J. and Horwath, P.H. (1996) T-Cell Cytokine Profile Evaluated as a Single Level in BAL and Blood in Allergic Asthma. American Journal of Respiratory Cell and Molecular Biology, 14, 319-326. http://dx.doi.org/10.1165/ajrcmb.14.4.8600935

[20] Till, S.J., Durham, S.R., Rajakulasingham, K., Humbert, M., Huston, D., Dickson, R., Kay, A.B. and Corringan, C.J. (1998) Allergen-Induced Proliferation and Interleukin-5 Production by Bronchoalveolar Lavage and Blood T Cells after Segmental Allergen Challenge. American Journal of Respiratory Cell and Molecular Biology, 158, 404-411. http://dx.doi.org/10.1164/ajrccm.158.2.9705007

[21] Umetsu, D.T., Akbari, O., DeKruyff, R.H., Shearer, W.T., Rosenwasser, L.J. and Bochner, B.S. (2003) Regulatory T Cells Control Development of Allergic Disease and Asthma. Journal of Allergy and Clinical Immunology, 112, 480-487. http://dx.doi.org/10.1016/S0091-6749(03)01869-4

[22] Magnan, A.O., Mely, L.G., Camilla, C.A., Badier, M.M., Montero-Julian, F.S., Guillot, C.M., Casano, B.B., Prato, S.J., Fert, V., Bongrand, P. and Vervloet, D. (2000) Assesssment of the Th1/Th2 Paradigm in Whole Blood in Atopy and Asthma. American Journal of Respiratory and Critical Care Medicine, 161, 1790-1796. http://dx.doi.org/10.1164/ajrccm.161.6.9906130

[23] Pelikan, Z., Pelikan-Filipek, M. and Oostenbrink, J.H. (1997) Delayed Asthmatic Response (DYAR), Its Clinical Features and Pharmacologic Modulation. Journal of Allergy and Clinical Immunology, 99, 321.

[24] Pelikan, Z. (2010) Delayed type of Asthmatic Response to Bronchial Challenge with Allergen, I: Clinical Features. Annals of Allergy, Asthma \& Immunology, 104, 394-404. http://dx.doi.org/10.1016/j.anai.2010.03.014

[25] Pelikan, Z. (2011) Delayed Asthmatic Response: A New Phenotype of Bronchial Response to Allergen Challenge and Soluble Adhesion Molecules in the Serum. Annals of Allergy, Asthma \& Immunology, 106, 119-130. http://dx.doi.org/10.1016/j.anai.2010.11.002

[26] Pelikan, Z. (2011) Delayed Asthmatic Response to Bronchial Challenge with Allergen-Mediators, Eicosanoids, Eosinophil and Neutrophil Constituents in the Blood and Urine. Respiration, 82, 225-236. http://dx.doi.org/10.1159/000324542

[27] Melillo, G., Bonini, S., Cocco, G., Davies, R.J., De Monchy, J.G.R., Frølund, L. and Pelikan, Z. (1997) Provocation Tests with Allergens. Allergy, 52, 5-35. http://dx.doi.org/10.1111/j.1398-9995.1997.tb04814.x

[28] Houd, D.J. and Taylor, C.C. (1987) Multivariate Analysis of Variance and Repeated Measures. Chapman and Hall, London.

[29] Härtel, C., Adam, N., Strunk, T., Temming, P., Müller-Steinhardt, M. and Schultz, C. (2005) Cytokine Responses Correlate Differentially with Age in Infancy and Early Childhood. Clinical and Experimental Immunology, 142, 446-453.

[30] Matsumoto, K., Gauvreau, G.M., Rerecich, T., Watson, R.M., Wood, L.J. and O’Byrne, P.M. (2002) IL-10 Production in Circulating T Cells Differs between Allergen-Induced Isolated Early and Dual Asthmatic Responders. Journal of Allergy and Clinical Immunology, 109, 281-286. http://dx.doi.org/10.1067/mai.2002.121144

[31] Kawayama, T., O’Byrne, P.M., Watson, R.M., Killian, K.J., Duong, M., Yoshida, M. and Gauvreau, G.M. (2006) Effects of Inhaled Ciclesonide on Circulating T-Helper Type 1/T-Helper Type 2 Cells in Atopic Asthmatics after Allergen Challenge. Clinical \& Experimental Allergy, 36, 1417-1424. http://dx.doi.org/10.1111/j.1365-2222.2006.02592.x

[32] Terashima, T., Amakawa, K., Matsumaru, A., van Eeden, S., Hogg, J.C. and Yamaguchi, K. (2001) BAL Induces an Increase in Peripheral Blood Neutrophils and Cytokine Levels in Healthy Volunteers and Patients with Pneumonia. Chest, 119, 1724-1729. http://dx.doi.org/10.1378/chest.119.6.1724

[33] Peek, E.J., Richards, D.F., Faith, A., Lavander, P., Lee, T.H., Corrigan, C. and Hawrylowitz, C.M. (2005) Interleukin-10Secreting "Regulatory” $\mathrm{T}$ Cells Induced by Glucocorticoids and $\beta 2$-Agonists. American Journal of Respiratory Cell and 
Molecular Biology, 33, 105-111. http://dx.doi.org/10.1165/rcmb.2005-0100OC

[34] Smithgall, M.D., Comeau, M.R., Park Yoon, B.R., Kaufman, D., Armitage, R. and Smith, D.E. (2008) IL-33 Amplifies both Th1- and Th2-Type Responses through Its Activity on Human Basophils, Allergen-Reactive Th2 Cells, iNKT and NK Cells. International Immunology, 20, 1019-1030. http://dx.doi.org/10.1093/intimm/dxn060

[35] Mathy, N.L., Scheuer, W., Lanzendörfer, M., Honold, K., Ambrosius, D., Norley, S. and Kurth, R. (2000) Interleukin-16 Stimulates the Expression and Production of Pro-Inflammatory Cytokines by Human Monocytes. Immunology, 100, 63-69. http://dx.doi.org/10.1046/j.1365-2567.2000.00997.x

[36] McFadden, C., Morgan, R., Rahangdale, S., Green, D., Yamasaki, H., Center, D. and Cruikshank, W. (2007) Preferential mIGRATION of T Regulatory Cells Induced by IL-16. Journal of Immunology, 179, 6439-6445.

[37] Strengell, M., Matikainen, S., Siren, J., Lehtonen, A., Foster, D., Julkunen, I. and Sareneva, T. (2003) IL-21 in Synergy with IL-15 or IL-18 Enhances IFN- $\gamma$ Production in Human NK and T Cells. Journal of Immunology, 170, 5464-5469.

[38] Kimura, M., Tsuruta, S. and Yoshida, T. (1999) Differences in Cytokine Production by Peripheral Blood Mononuclear Cells (PBMC) between Patients with Atopic Dermatitis and Bronchial Asthma. Clinical \& Experimental Immunology, 118, 192-196. http://dx.doi.org/10.1046/j.1365-2249.1999.01055.x

[39] Varga, E.M., Wachholtz, P., Nouri-Aria, K.T., Verhoef, A., Corrigan, C.J., Till, S.J. and Durham, S.R. (2000) T Cells from Human Allergen-Induced Late Sthmatic Responses Express IL-12 Receptor $\beta 2$ Subunit mRNA and Respond to IL-12 in Vitro. Journal of Immunology, 165, 2877-2885.

[40] Moverare, R., Elfman, L., Moverare, R., Stalenheim, G. and Björnsson, E. (2000) Study of the Th1/Th2 Balance, Including IL-10 Production, in Cultures of Peripheral Blood Mononuclear Cells from Birch-Pollen-Allergic Patients. Allergy, 55, 171-175. http://dx.doi.org/10.1034/j.1398-9995.2000.00244.x

[41] Lim, S., John, M., Seybold, J., Taylor, D., Witt, C., Barnes, P.J. and Chung, K.F. (2000) Increased Interleukin-10 and Macrophage Inflammatory Protein-1 $\alpha$ Release from Blood Monocytes ex Vivo during Late-Phase Response to Allergen in Asthma. Allergy, 55, 489-495. http://dx.doi.org/10.1034/j.1398-9995.2000.00483.x

[42] Contreras, J.P., Ly, N.P., Gold, D.R., He, H., Wand, M., Weiss, S.T., Perkins, D.L., Platts-Mills, T.A.E. and Finn, P.W. (2003) Allergen-Induced Cytokine Production, Atopic Disease, IgE and Wheeze in Children. Journal of Allergy and Clinical Immunology, 112, 1072-1077. http://dx.doi.org/10.1016/j.jaci.2003.08.036

[43] Böttcher, M.F., Bjurström, J., Mai, X.M., Nilsson, L. and Jenmalm, M.C. (2003) Allergen-Induced Cytokine Secretion in Atopic and Non-Atopic Asthmatic Children. Pediatric Allergy and Immunology, 14, 345-350. http://dx.doi.org/10.1034/j.1399-3038.2003.00061.x

[44] John, M., Lim, S., Seybold, J., Jose, P., Robichaud, A., O’Connor, B., Barnes, P.J. and Chung, K.F. (1998) Inhaled Corticosteroids Increase Interleukin-10 but Reduce Macrophage Inflammatory Protein-1 $\alpha$, Granulocyte-Macrophage Colony Stimulating Factor and Interferon- $\gamma$ Release from Alveolar Macrophages in Asthma. American Journal of Respiratory and Critical Care Medicine, 157, 256-262. http://dx.doi.org/10.1164/ajrccm.157.1.9703079

[45] Noma, T., Sugawara, Y., Ogawa, N., Saeki, T., Yamaguchi, K. and Kawano, Y. (2004) Dermatophagoides-Induced Interleukin-10 Production by Peripheral Blood Lymphocytes from Patients with Asthma in Remission. Pediatric Allergy and Immunology, 15, 459-468. http://dx.doi.org/10.1111/j.1399-3038.2004.00176.X

[46] Pinsonneault, S., El Bassam, S., Mazer, B., Cruikshank, W. and Laberge, S. (2001) IL-16 Inhibits IL-5 Production by Antigen-Stimulated T Cells in Atopic Subjects. Journal of Allergy and Clinical Immunology, 107, 477-482. http://dx.doi.org/10.1067/mai.2001.112373

[47] Xystrakis, E., Kusumakar, S., Boswell, S., Peek, E., Urry, Z., Richards, D.F., Adikibi, T., Pridgeon, C., Dallman, M., Loke, T.K., Robinson, D.S., Barrat, F.J., O’Garra, A., Lavender, P., Lee, T.H., Corrigan, C. and Hawrylowicz, C.M. (2006) Reversing the Defective Induction o f IL-10-Secreting Regulatory T Cells in Glucocorticoid-Resistant Asthma Patients. Journal of Clinical Investigation, 116, 146-155. http://dx.doi.org/10.1172/JCI21759

[48] Clough, J.B., Keeping, K.A., Edwards, L.C., Freeman, W.M., Warner, J.A. and Warner, J.O. (1999) Can We Predict Which Wheezy Infants Will Continue to Wheeze? American Journal of Respiratory and Critical Care Medicine, 160, 1473-1480. http://dx.doi.org/10.1164/ajrccm.160.5.9807019

[49] Lenarczyk, A., Helsloot, J., Farmer, K., Peters, L., Sturgess, A. and Kirkham, B. (2000) Antigen-Induced IL-17 Response in the Peripheral Blood Mononuclear Cells (PBMC) of Healthy Controls. Clinical \& Experimental Immunology, 122, 41-48. http://dx.doi.org/10.1046/j.1365-2249.2000.01328.x

[50] Thunberg, S., Gafvelin, G., Nord, M., Grönneberg, R., Grunewald, J., Eklund, A. and van Hage, M. (2010) Allergen Provocation Increases Th2-Cytokines and FOXP3 Expression in the Asthmatic Lungs. Allergy, 65, 311-318. http://dx.doi.org/10.1111/j.1398-9995.2009.02218.x

[51] El Bassam, S., Pinsonneault, S., Kornfeld, H., Ren, F., Menezes, J. and Lagerge, S. (2006) Interleukin-16 Inhibits Interleukin-13 Production by Allergen-Stimulated Blood Mononuclear Cells. Immunology, 117, 89-96. http://dx.doi.org/10.1111/j.1365-2567.2005.02269.x 
[52] Jenmalm, M.C., Van Snick, J., Cormont, F. and Salman, B. (2001) Allergen-Induced Th1 and Th2 Cytokine Secretion in Relation to Specific Allergen Sensitization and Atopic Symptoms in Children. Clinical \& Experimental Allergy, 31, 1528-1535. http://dx.doi.org/10.1046/j.1365-2222.2001.01190.x

[53] Pelikan, Z. (2012) Delayed Type of Asthmatic Response to Allergen Challenge and Cytokines in the Peripheral Blood. Respiration, 84, 385-395. http://dx.doi.org/10.1159/000335258

[54] Sterk, P.J., Fabbri, L.M., Quanjer, Ph.H., Cockroft, D.W., O’Byrne, P.M., Anderson, S.D., Juniper, J.F. and Malo, J.L. (1993) Airway Responsiveness-Standardized Challenge Testing with Pharmacological, Physical and Sensitizing Stimuli in Adults. European Respiratory Journal, 6, 53-83. http://dx.doi.org/10.1183/09041950.053s1693

[55] Postma, D.S. and Boezen, H.M. (2004) Rationale of the Dutch Hypothesis. Allergy and Airway Hyperresponsiveness as Genetic Factors and Their Interaction with Environment in the Development of Asthma and COPD. Chest, 126, 96S-104S. 